\title{
Investigation of background sources of muography
}

\section{László Oláh*}

Earthquake Research Institute, The University of Tokyo

E-mail: olah@eri.u-tokyo.ac.jp

\section{Hiroyuki K. M. Tanaka}

Earthquake Research Institute, The University of Tokyo

E-mail: ht@eri.u-tokyo.ac.jp

\section{Dezső Varga}

Wigner Research Centre for Physics of the Hungarian Academy of Sciences

E-mail: varga.dezso@wigner.mta.hu

\begin{abstract}
Muography is a technique for the imaging of the interior of large size objects, such as volcanoes, mountains, with the measurement of the flux of high energy cosmic muons. The electrons/positrons, high energy hadrons and low energy muons can contaminate the muon signal. These background sources require a good understanding of the creation processes, as well as reliable simulation frameworks with high predictive power. A simulation framework based on GEANT4 has been established to pin down the key features of the background particles. The particle spectra and ratios have been compared to existing other measurements as well as other simulation programs. The correlation between simultaneously arriving particles have also been quantitatively investigated, demonstrating that electrons and positrons tend to arrive close to each other and with low relative angle. This feature, which is highly relevant for counting detectors, has been experimentally verified at shallow depth underground and under open sky using the new variant of MWPC detectors. A low-noise MWPC-based tracking detector (Muographic Observation System, mMOS) has been installed at the Sakurajima volcano. The report on the detector performance and the verification of the applicability of mMOS for long-range muography will be presented.
\end{abstract}

35th International Cosmic Ray Conference - ICRC2017 -

10-20 July, 2017

Bexco, Busan, Korea

\footnotetext{
* Speaker.
} 


\section{Introduction}

The primary cosmic rays isotropically enter the Earth's atmosphere and produce mesons via hadronic interactions. The competitive energy dependent processes, e. g. the decays and interactions produce the muonic-, soft-, and hadronic components of the cosmic radiation. Beside the three components, the very high energy $(>\mathrm{TeV}$ ) primary cosmic rays create extensive air showers which spread over large area at the surface of the Earth.

The cosmic-ray muons can penetrate across a large amount of material (kiometers of rock) due to their relatively high energy, as well as the lack of hadronic interactions and bremsstrahlung radiation. Muons continually loss from their energy in the material and they are absorbed if their initial energy less then the value which is necessary the penetrate the material. The total energy loss of muons depends on the density-length (average density $\times$ thickness) of the material. Thus one can determine the average density (thickness) of the material with the knowledge of its thickness (density) and the measurement of the flux of muons behind the material. This imaging technique is called muography. Tomographic imaging can also be achieved with measurements which are performed around the target of interest from different directions.

The applicability of muography was demonstrated in various interdisciplinary fields, such as for searching hidden caverns inside historical buildings [1], above underground cavern and tunnel systems [2], for imaging [3] and monitoring [4] the density variations inside active volcanoes.

The low energy muons, the high energy electrons/positrons and protons are also detected during muographic observations by each type of particle detector (mostly scintillators, emulsions and gaseous detectors are used) under open sky. These muons and electrons are produced by the interactions of hadrons in the atmosphere or in the near surface of the target of interest. These background particles scatter inside the detector from the same direction as the high energy muons which penetrated across the target of interest. In addition, upward-going and backward-going particles are also observed. In Ref. [5], the energy cut of about $1 \mathrm{GeV}$ was suggested for particle detectors to suppress background particles. The nature of background depends on the locations of the target of interest and the particle detector [5]. The study of the background of muograpic observations requires a dedicated simulation framework which produces correlated cosmic particle showers, as well as the investigated object and the particle detectors can be implemented into its framework.

Present paper is organized as follows. In Sec. 2, we introduce our GEANT4-based simulation framework for the investigation of background particles in muograpic observations. Section 3 and 4 focus on the results on the origin and nature of background particles under open sky and at underground. In Sec. 5, we present the MWPC-based Muographic Observation System (mMOS) and its first test measurements at the Sakurajima volcano, Kyusu, Japan.

\section{A GEANT4-based simulation framework for study of background particles}

The GEANT4.9.4.p01 [6] release is applied to simulate the penetration of cosmic particles across the Earth's atmosphere and take into account the detector effects [7]. The simulation incorporates all of the relevant electromagnetic and hadronic physics processes as well as the magnetic field of the Earth can also be implemented. The atmosphere is modeled by a $5 \times 5 \times 10 \mathrm{~km}^{3}$ rectangular volume which is horizontally divided into 20 layers. Each layer is composed from $\mathrm{N}_{2}, \mathrm{O}_{2}$, 
and $\mathrm{Ar}$ with the volume fractions of $78.1 \%, 21.0 \%$ and $0.9 \%$, respectively. The main parameters of the simulation are the type of "primary" particles (primary for the simulation and not the true primary cosmic rays), altitude of production, exponent of energy distribution and zenith angle. To simulate the detector effects, 10 layers of low material budget (thickness of $2 \mathrm{~mm}$, density of 1.7 $\mathrm{gcm}^{-3}$ ) detectors [8], made from printed circuit board, are deployed parallel under each other with equivalent distance of $5 \mathrm{~cm}$ at sea level.

The primary particles are injected from the top of the atmosphere with power low energy distribution with the exponent of -2.7. The passage of primary and secondary charged particles are tracked across to atmosphere and the detector down to sea level. The ID numbers, energy, momentum and position vectors of charged particles are recorded. Finally, an event-by-event analysis was performed on the simulated data to extract their spectra, ratios, and correlations. The detailed description of the simulation framework is presented in Ref. [7]. In the following sections the results of simulations are presented, interpreted and compared to the experimental observations.

\section{Sources of soft component}

The soft component originates from well understood physics processes in the atmosphere. Energetic electrons and positrons are produced by decay of muons, the resulting charge ratio follows the charge ratio of muons. Gammas are mostly created by Bremmstrahlung. The gammas creates electrons and positrons via pair production. Electromagnetic shower is produced by periodic recurrence of the two previous processes. Ionizations and Compton scattering are also produce electrons. Decays of hadrons contribute also to electrons and gammas in a small fraction because of their low abundance.

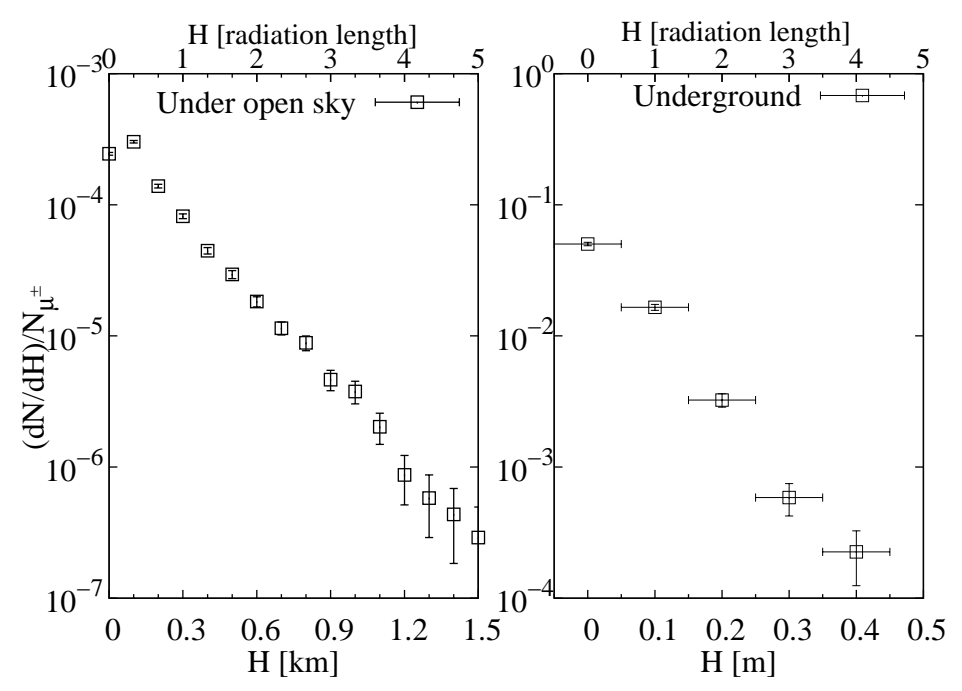

Figure 1: The creation altitude of the detected electrons under open sky at sea level (left panel) and at the depth of 50 meter-standard-rock-equivalent (right panel) [7]. 
At underground, the detected soft particles are created by the ionization of muons and electrons, pair production of gammas and Compton scattering of electrons. The decay of muons does not contribute to the detected soft particles [7].

The soft particles travel the distance of few radiation lengths before a new step of the cascade process takes place. Figure 1 shows the number of detected electrons $(N)$ as a function of creation altitude $(H)$ under open sky at sea level (left panel) and at the depth of 50 meter-standard-rockequivalent (right panel) [7]. The interpretation of the results is the following: the production of the soft particles is a dynamic process, where at the characteristic distance is the radiation length, and the particles are created or absorbed, as well as continuously reproduce the number distributions at each altitude above the observation level. The observed distributions largely lost the dependence on the initial conditions.

The particle spectra and ratios were also produced by the developed simulation framework and all of them were in good agreement with the experimental observations, other simulations and calculations [7].

\section{Spatial and direction correlations}

In case of real-time muographic observations, the simultaneous appearance of particles becomes important. The simultaneously detected particles originate from the same shower. Since these are relativistic particles, the difference between their arrival time is in the order of few nanoseconds, and the expected distance between them is in order of few meters under open sky.

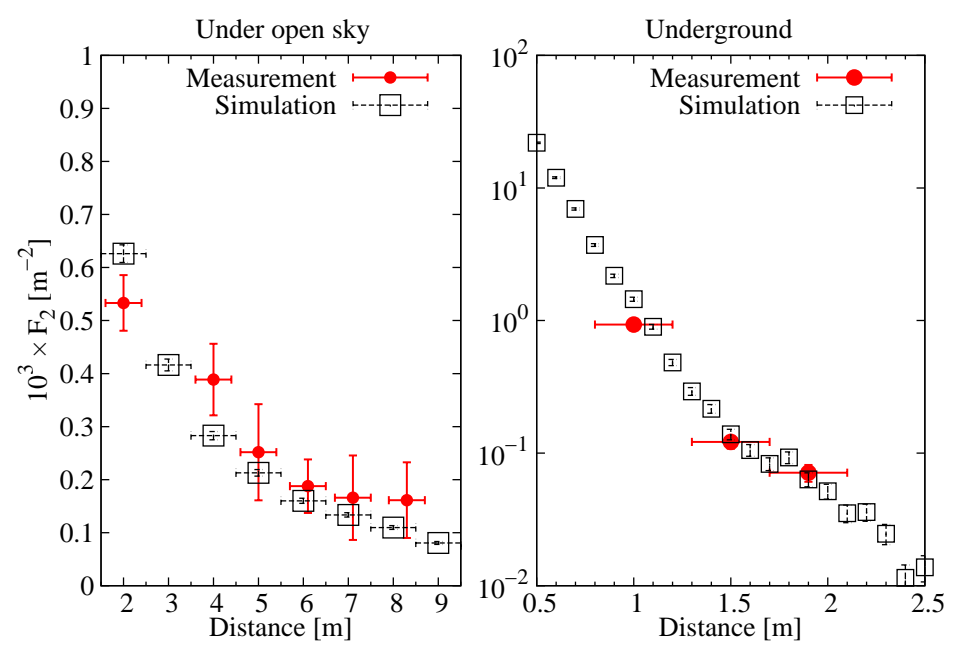

Figure 2: The flux of particle pairs, $\mathrm{F}_{2}$ as a function of their distance under open sky (left panel) and at 7.5 meter depth underground (right panel). The experimental observations (red dots) are in agreement with the simulations (empty rectangles) [7]. 
The so-called "two particle flux", $F_{2}$, is defined as

$$
F_{2}=\frac{\mathrm{d} N_{12}}{2 \pi R \mathrm{~d} R} \frac{1}{N_{1}},
$$

where $N_{12}$ is the number of pairs with the distance of $R, 2 \pi R \mathrm{~d} R$ is the area of the ring with the radius of $R$ and with the width of $\mathrm{d} R$ and $N_{1}$ being the number of all particles. One can refer to $N_{1}$ as "triggers", and then $N_{12}$ is the number of correlated particles in coincidence with the trigger. Due to symmetric considerations, the trigger particle to be at the origin and the correlated particle at a distance of $R$ (or inversely).

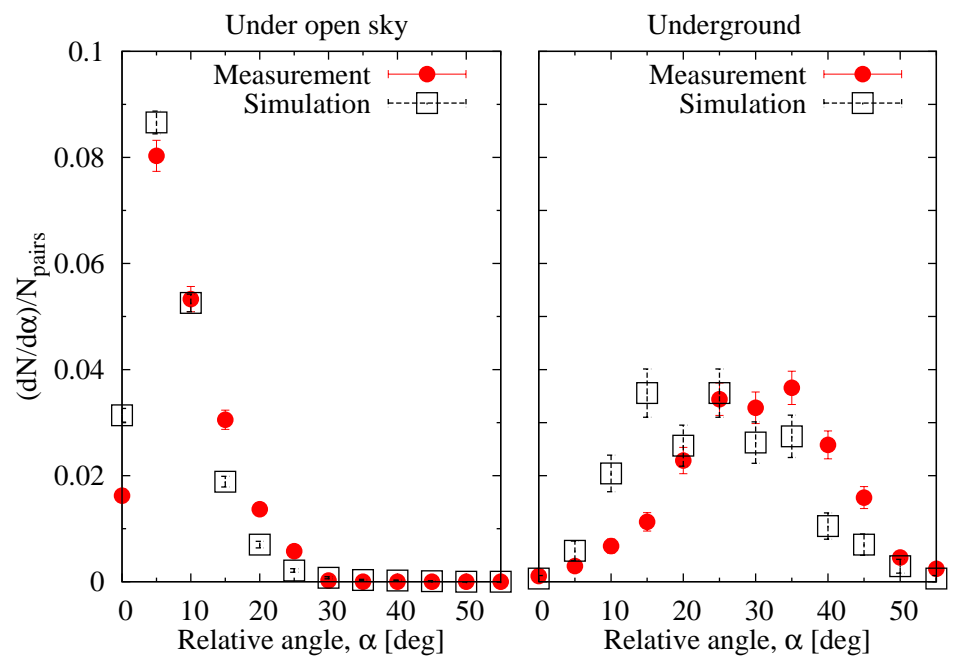

Figure 3: The distribution of relative angles $(\alpha)$ of particle pairs which arrive within the distance of $2 \mathrm{~m}$ under open sky (left panel) and at 7.5 meter depth underground (right panel). The measurements are in reasonable agreement with the simulations [7].

The "two particle flux", $F_{2}$ was determined by the simulations framework as well as by our measurements [7]. The measurements were performed by two MWPC-based tracking detectors, consist of four layers each. The so called master detector triggered the measurements and other was the slave which measured the correlated particles. Different measurements were performed under open sky and at $7.5 \mathrm{~m}$ depth underground up to the distance of 8.3 meter. The complete descriptions of the experiments are presented in Ref. [7]. Figure 2 shows two particle fluxes as a function of distance under open sky (left panel) and at underground (right panel). Figure 3 shows the distribution of relative angles of particle pairs which arrive within the distance of $2 \mathrm{~m}$ under open sky (left panel) and at the depth of $7.5 \mathrm{~m}$ right panel. Note that the underground relative angle distribution confirms that the detected particles are created at the last few radiation lengths $(<50 \mathrm{~cm})$ above the observation level. Both of the simulation (red dots) and the experimental results (empty black rectangles) confirm that the particle pairs are detected within small distances $(<10 \mathrm{~m})$ and with a small relative angles. Furthermore, these results demonstrate that the proposed simulation framework can reproduce the spatial and directional correlations of cosmic showers. 


\section{A high precision MWPC-based Muographic Observation System}

A lightweight, high precision, MWPC-based muographic observation system (mMOS) was designed for muographic observations as part of the collaboration between the Wigner Research Centre for Physics of the HAS and the Earthquake Research Institute, the University of Tokyo. This system is based on a new variant of multi-wire proportional chambers (MWPC) [9]. In January 2017, the first prototype of the MWPC-based Muographic Observation System was installed at the foot of the Sakurajima volcano in Kyushu, Japan, and was oriented to the direction of Showacrater, its most active crater which erupted more than 1000 times in 2015. The distance between the mMOS and the Showa-crater was $2.8 \mathrm{~km}$. To suppress the background particles, the mMOS consists of seven MWPCs with a size of $0.8 \times 0.8 \mathrm{~m}^{2}$ and five identically sized lead absorbers with a thickness of $2 \mathrm{~cm}$ each. The total length of the tracking system is $2 \mathrm{~m}$ and since there is a reasonable position resolution of $4 \mathrm{~mm}$ in both dimensions, an angular resolution of at least $10 \times 10 \mathrm{mrad}^{2}$ can be achieved by the mMOS. This relatively good angular resolution allows us to image the interior of Showa-crater with a precision of $30 \times 30 \mathrm{~m}^{2}$.

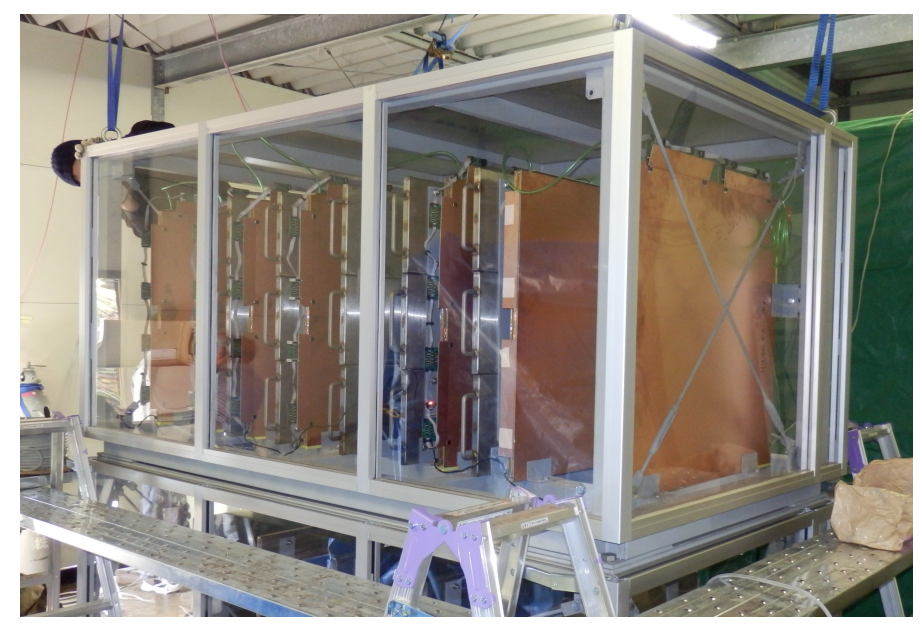

Figure 4: The photo of the first prototype of the MWPC-based Muographic Observation System (mMOS), which is installed at the Sakurajima volcano, Kyusu, Japan.

Figure 4 shows the photo of mMOS which was housed in steel frame with plexi-glass walls. The MWPCs are supplied with the environmental friendly $\mathrm{Ar}-\mathrm{CO}_{2}$ (80:20) gas mixture which continuously flows across the mMOS with the flow of about 1-2 liters per hour [8,9]. The DAQ and front-end electronics are controlled by a Rasberry Pi computer [9, 10], remote access and control are provided via Virtual Private Network. The power consumption of the complete tracking system is about 5 Watt. The mMOS reliably operated during the first half year of data taking with the tracking efficiency of above $95 \%$. Detector maintenance was performed two times during the time of data taking to replace the gas bottle.

To demonstrate that the mMOS is applicable to high-precision muography with low backgroundnoise, we compared the measured flux to the expected one. This latter flux was determined as follows. First of all, the thickness of the volcano was measured and calculated to the coordinate system of the detector in $10 \times 10 \mathrm{mrad}^{2}$ angular bins. The threshold energy which is necessary 
for cosmic muons to penetrate the volcano was calculated for each angular bin. The volcano was assumed to $\mathrm{SiO}_{2}$ with the density of $2 \mathrm{gcm}^{-3}$. Finally, empirical muon spectra based on the Modified Gaisser Model [11] was integrated from the energy threshold to $10 \mathrm{TeV}$ for each angular bin. Figure 5 shows the comparison of the measured flux (red dots with $1 \sigma$ errorbars) and the expected flux (solid black line) as a function vertical (elevation) angle between the azimuth angles of \pm 40 mrad. The fluxes show a reasonable agreement at the upper part of the volcano. One can conclude that the mMOS sufficiently suppresses the background particles and measures precisely the flux of cosmic muons.

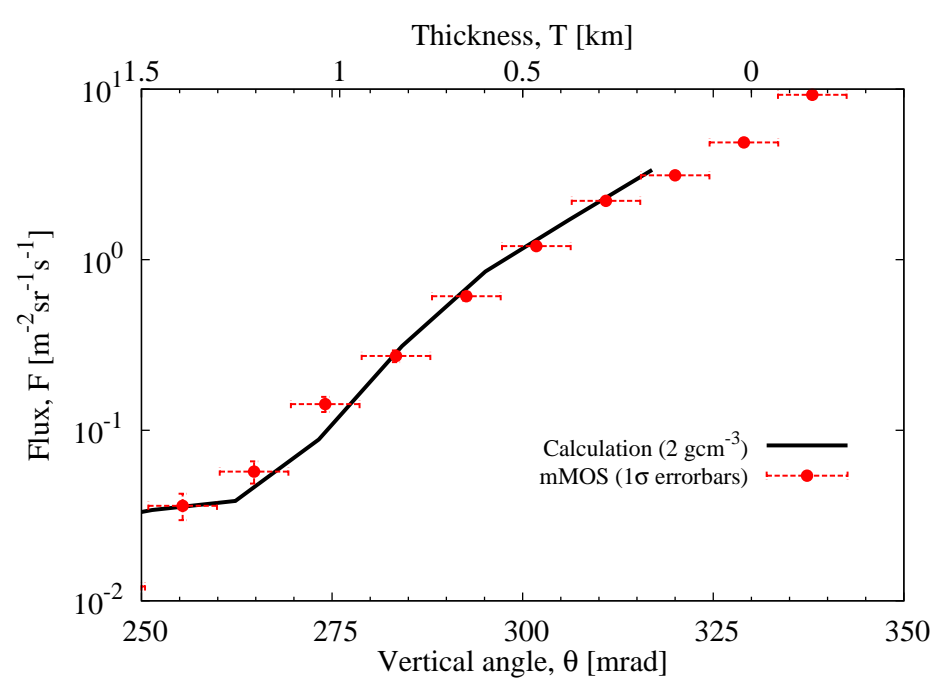

Figure 5: The comparison of the expected (solid black line) and measured (filled red circles) fluxes demonstrates that the mMOS suppresses the background particles and measures precisely the flux of cosmic muons.

The development of a real-time monitoring system is ongoing and the extension of the detector surface to about $10 \mathrm{~m}^{2}$ is planned during the next years. With that detector surface statistically significant muographs can be achieved within the time intervals of 6-12 hours and the muography can become the part of a forecast system together with the ordinary geophysical methods.

\section{Summary}

Muographic observations require the suppression of low energy muons and high energy soft particles and hadrons. A GEANT4-based simulation framework has been developed for the investigation of sources and basic features of background particles. We found that the soft particles originate from the last few radiation lengths above the detector level and arrive simultaneously within a low relative angle and small distance $(<10 \mathrm{~m})$. The results are qualitatively the same at underground as under open sky, except that the characteristic distances are shorter due to the smaller radiation length. The presented simple muon initiated simulations can reproduce the development of correlated showers and basic features of soft component. In addition, the model of 
muography observations can be implemented in this framework and the background sources can be explored. A light weight, high precision MWPC-based Muographic Observation System has been developed. The first test of the mMOS has been performed at Sakurajima volcano, Kyusu, Japan. We demonstrated that the tracking system suppresses the background particles and measures precisely the flux of cosmic muons.

\section{Acknowledgement}

The authors acknowledge the support of the "Momentum" Grant of the Hungarian Academy of Sciences under the No. LP2013-60, the MEXT Integrated Program for the Next Generation Volcano Research and Human Resource Development, as well as the members of the REGARD group.

\section{References}

[1] L. W. Alvarez et al.: Search for hidden chambers in the pyramids, Science 167 (1970) 832-839

[2] L. Oláh et al.: CCC-based muon telescope for examination of natural caves, Geosci. Instrum. Method. Data Syst. 1 (2012) 229-234

[3] H. K. M. Tanaka et al.: High resolution imaging in the inhomogeneous crust with cosmic-ray muon radiography: The density structure below the volcanic crater floor of Mt. Asama, Japan, Earth and Planetary Science Letters 263 (2007) 104-113

[4] H. K. M. Tanaka et al.: Radiographic visualization of magma dynamics in an erupting volcano, Nat. Commun. 5:3381 doi: 10.1038/ncomms4381 (2014)

[5] R. Nishiyama et al.: Monte Carlo simulation for background study of geophysical inspection with cosmic-ray muons, Geophysical Journal International 206 (2016) 1039-1050

[6] S. Agostinelli et al.: Geant4 - a simulation toolkit, Nucl. Instrum. Meth. A 506 (2003) 250-303

[7] L. Oláh and D. Varga: Investigation of soft component in cosmic ray detection, Astroparticle Physics 93 (2017) $17-27$

[8] L. Oláh et al.: Cosmic Muon Detection for Geophysical Applications, Advances in High Energy Physics 2013 (2013) 560192

[9] D. Varga et al.: High Efficiency Gaseous Tracking Detector for Cosmic Muon Radiography, Advances in High Energy Physics 2016 (2016) 1962317

[10] L. Oláh et al.: Close cathode chamber technology for cosmic particle tracking, Journal of Physics: Conference Series 632 (2015) 012020

[11] Alfred Tang et al.: Muon simulations for Super-Kamiokande, KamLAND, and CHOOZ, Phys. Rev. D 74 (2006) 053007 